Plasma Interactions in Laser Irradiated Semi-Cylindrical Cavities Studied with Soft X-Ray Interferometry Using a Capillary Discharge Laser

M. A. Purvis, J. Grava, J. Filevich, M.C. Marconi, J. J. Rocca, S. J. Moon, J. Dunn, J. Nilsen, V. N. Shlyaptsev, E. Jankowska

September 25, 2007

The International Society for Optical Engineering Soft X-Ray Lasers and Applications VII 
This document was prepared as an account of work sponsored by an agency of the United States government. Neither the United States government nor Lawrence Livermore National Security, LLC, nor any of their employees makes any warranty, expressed or implied, or assumes any legal liability or responsibility for the accuracy, completeness, or usefulness of any information, apparatus, product, or process disclosed, or represents that its use would not infringe privately owned rights. Reference herein to any specific commercial product, process, or service by trade name, trademark, manufacturer, or otherwise does not necessarily constitute or imply its endorsement, recommendation, or favoring by the United States government or Lawrence Livermore National Security, LLC. The views and opinions of authors expressed herein do not necessarily state or reflect those of the United States government or Lawrence Livermore National Security, LLC, and shall not be used for advertising or product endorsement purposes. 


\title{
Plasma interactions in laser irradiated semi-cylindrical cavities studied with soft $x$-ray interferometry using a capillary discharge laser
}

\author{
M. Purvis, J. Grava, J. Filevich, M.C. Marconi, J.J. Rocca \\ NSF ERC for Extreme Ultraviolet Science and Technology and Department of Electrical and \\ Computer Engineering, Colorado State University, Fort Collins, Colorado 80523 \\ James Dunn, Stephen J. Moon, Joseph Nilsen \\ Lawrence Livermore National Laboratory, Livermore, California 94551, USA \\ Vyacheslav N. Shlyaptsev \\ Department of Applied Science, University of California Davis-Livermore, Livermore, California \\ 94551, USA \\ Elizabeth Jankowska \\ Wroclaw University of Technology, Wroclaw, Poland
}

\begin{abstract}
Soft x-ray interferometry was used to measure the evolution of dense converging plasmas created by laser irradiation of $500 \mu \mathrm{m}$ diameter semi-cylindrical carbon targets. Optical laser pulses with an intensity of $\sim 1 \times 10^{12} \mathrm{~W} \mathrm{~cm}{ }^{-2}$ and $120 \mathrm{ps}$ duration were used to heat the surface of the cavities. The dense plasma formed expands from the walls converging slightly off the semi-cylinder's axis, giving rise to a bright localized high density plasma region. A sequence of electron density maps were measured using a $46.9 \mathrm{~nm}$ wavelength tabletop capillary discharge soft x-ray laser probe and a amplitude division interferometer based on diffraction gratings. The measured density profiles are compared with simulations conducted using the multi-dimensional hydrodynamic code HYDRA. The benchmarked model was then used to simulate particle trajectories which reveal that the increase in electron density near the axis is mainly the result of the convergence of plasma that originated at the bottom of the groove during laser irradiation.
\end{abstract}

Keyword list: soft x-ray interferometry, soft x-ray lasers, dense plasma diagnostics, hydrodynamics simulations

\section{INTRODUCTION}

The dynamics of $2 \mathrm{D}$ colliding plasmas are relevant to both laboratory plasmas as well as to the much larger astrophysical plasma phenomena [1]. Laser-created laboratory plasma experiments can be used to study phenomena, such as shocks, radiation diffusion, and polar jets at a greatly reduced scale $[1,2,3]$. Plasma collisions in cylindrical cavities are a concern for indirect drive inertial confinement fusion $[4,5]$ where laser irradiation of hohlraums creates large pressure gradients that radially accelerate the plasma towards the axis where it collides. The collision dynamics depends on the degree of collisionality, which can range from stagnation to extended interpenetration [6]. Comparison of direct measurements of the electron density evolution with code simulations can contribute to a better understanding of the plasma dynamics, are important to benchmark complex codes and determine their regimes of validity.

The reduction of the probe wavelength increases the maximum density and plasma scale lengths that can be measured with plasma interferometry. This fact motivates the development of dense plasma diagnostic techniques based 
on soft x-ray laser probes. Wan et al. performed an interferometry experiment using a soft x-ray laser probe at $\lambda=15.5 \mathrm{~nm}$ to study colliding plasmas, created by irradiating two Au slabs aligned at $45^{\circ}$ with respect to the heating pulse axis, the target slabs were separated by a variable gap between the tips of the slabs [7]. Experimentally it was observed that as the plasma evolved, the collisionality changed from early time interpenetration into later time stagnation. The low repetition rate at which the experiment could be performed allowed only the acquisition of interferograms at a few instances during the plasma evolution. A comparison was made with the Lagrangian hydrodynamic code LASNEX. It was reported that the code predicted shock heated electron density peaks located off the symmetry plane, which were not observed in the experiment.

In the work reported in this paper we have used soft x-ray laser interferometry to obtain sequences of detailed plasma density maps of dense colliding plasmas, created by irradiating semi-cylindrical carbon cavities. We have, for the first time to our knowledge, determined the region of validity of soft $\mathrm{x}$-ray laser plasma interferograms by taking into account the contribution of bound electrons to the index of refraction. The plasmas were probed with the $\lambda=46.9 \mathrm{~nm}$ beam from a compact capillary discharge soft x-ray laser (SXRL) [8] using a high throughput interferometer that uses diffraction gratings to split and recombine the beam [9]. The high repetition rate of the capillary discharge laser allowed us to map the entire evolution of carbon plasmas with numerous high contrast soft x-ray laser interferograms. The density maps obtained from these interferograms describe the radial expansion of the plasma towards the axis of the cavity, where a dramatic increase in the electron density is observed. Comparison of the data with 2-dimensional (2D) simulations performed using the hydrodynamic code HYDRA show a very good agreement, indicating that the plasmas are highly collisional. A simulated tracer particle trajectory technique helped determine that the density increase on axis is dominantly caused by plasma converging from a region located at the back of the target surface. This paper starts with a description of the experimental set up, followed by a summary of the interferometry results, and a comparison of the experimental measurements with simulations. The results describe the dynamics responsible for the formation of an electron density build-up in converging plasmas expanding from semi-cylindrical cavities. Future work on the study of plasma expansion off "V shaped" targets under similar irradiation conditions, are briefly discussed.

\section{EXPERIMENTAL SETUP}

The plasmas were created by irradiating semi-cylinder cavities at intensities of $1 \times 10^{12} \mathrm{Wcm}^{-2}$ with $\lambda=800 \mathrm{~nm}$ pulses of 120 ps (FWHM) duration from a Ti:Sapphire laser. The target geometry is schematically illustrated in Fig. 1. Sets of half-cylinder grooves, $500 \mu \mathrm{m}$ in diameter, were machined on the edge of a $1 \mathrm{~mm}$ wide carbon slab with a spacing of $1 \mathrm{~mm}$ between grooves. A single slab comprised of 35 accessible grooves, allowing numerous interferograms to be obtained without having to vent the target chamber. The optical laser beam irradiating the cavity impinged into it at normal incidence with respect to the flat front surface of the target and the SXRL probed the plasma along the axis of the semi-cylinder cavity.

A complete diagram of the experimental setup can be found in a previous publication [10]. The $650 \mathrm{~mJ}$ laser pulses used to create the plasma were produced by a Ti:Sapphire laser consisting of a Kerr mode-locked oscillator and three stages of amplification, yielding pulses of $120 \mathrm{ps}$ duration. The relatively short pulse duration allows the rapid deposition of laser energy, making it possible to study the evolution of the plasma in the cavity without further laser heating of the plasma. The laser heating pulse was measured to be free of significant pre-pulses, i.e. no pre-pulses with intensities above the target ablation threshold. A vacuum spatial filter was used to smooth the laser beam profile and improve the uniformity of the laser energy deposition onto the target. The laser pulses were focused to form a line of 300 $\mu \mathrm{m}$ FWHM diameter and $1.5 \mathrm{~mm}$ FWHM length onto the target. The homogeneity of the line focus was monitored on every shot by imaging it on an equivalent focal plane. 


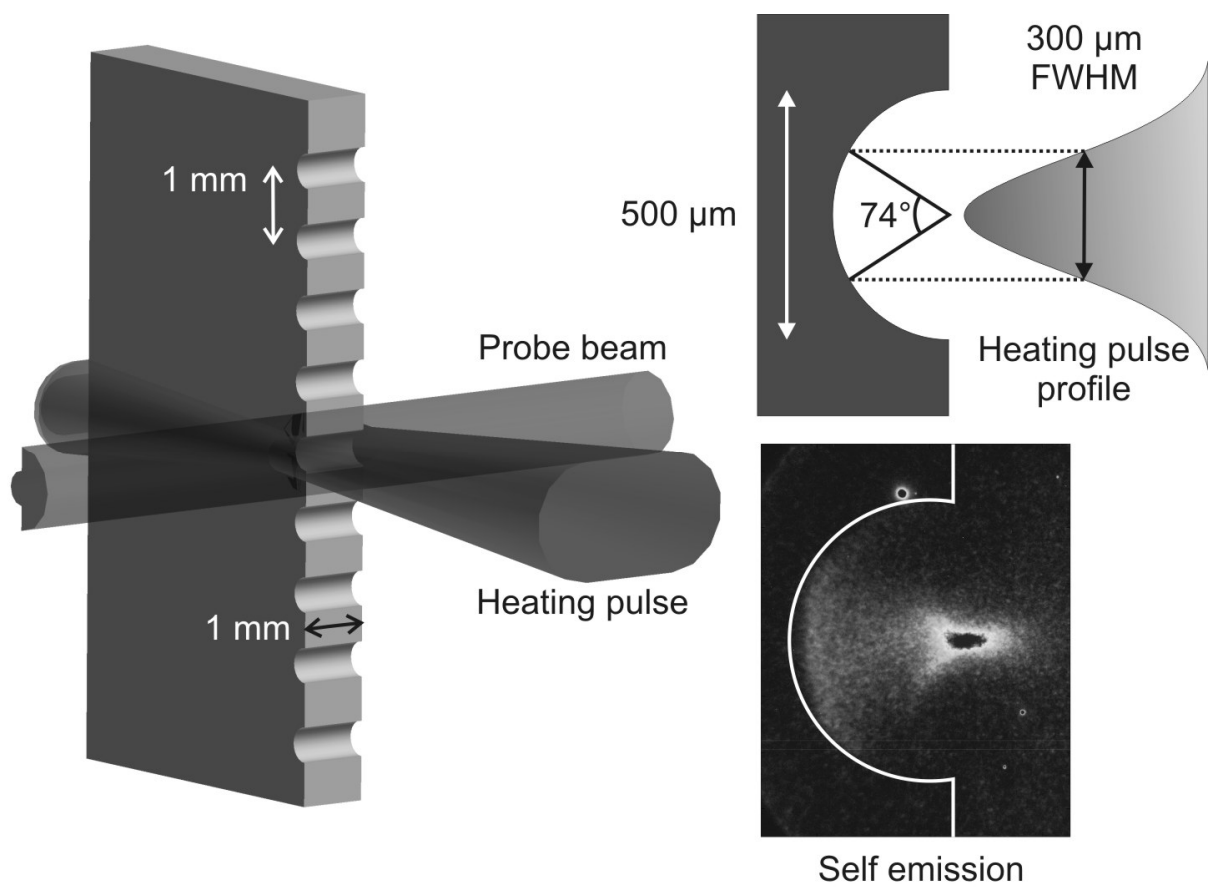

Figure 1. 3D representation of the target with the $\lambda=46.9 \mathrm{~nm}$ probe laser's path and the $\lambda=800 \mathrm{~nm}$, heating laser's path. The inset figure illustrates the $300 \mu \mathrm{m}$ FWHM Gaussian profile of the heating beam impinging onto the $500 \mu \mathrm{m}$ diameter cavity. The FWHM of the beam, which contains $77 \%$ of the energy, heats the target surface contained within an angle of 74 degrees. A time integrated image of the plasma self emission is shown bottom right.

The soft x-ray laser probe beam was generated by a high repetition rate table-top $46.9 \mathrm{~nm}$ Ne-like Ar capillary discharge laser $[8,11]$ that delivers pulses of $\sim 1 \mathrm{~ns}$ duration and $\sim 0.15 \mathrm{~mJ}$ of energy. The inherent jitter associated with the spark gap switch that controls the firing of the capillary discharge was reduced to $\sim 2 \mathrm{~ns}$ by a laser trigger scheme. This was achieved by focusing $30 \mathrm{~mJ}$ pulses from a Nd:YAG laser onto one electrode of the spark gap where it created a plasma initiating the breakdown of the switch. The entire evolution of the plasma could be mapped using 30-40 interferograms, with a single target strip.

The good spatial coherence of this laser assists in the generation of interferograms with high fringe visibility [12] when combined with an amplitude division soft x-ray interferometer developed at Colorado State University. The instrument is assembled in a skewed Mach-Zehnder geometry in which both the beam splitters and the reflecting mirrors are placed at grazing incidence for increased reflectivity. Ruled diffraction gratings with gold coatings are used as beam splitters. The gratings contribute to the robustness of the design. The blaze angle of the gratings is selected to direct equal intensity of the beam into the zero and first order. The instrument has a throughput of $6 \%$ per arm. More details on the interferometer and its alignment procedure can be found in previous publications $[9,13]$. The target was placed along the zero order path of the interferometer between the grazing incidence gold mirror and the second diffraction grating.

The timings between the probe pulse and the plasma heating pulse were measured using two photodiodes. The heating laser pulse was measured using a fast photodiode with a rise time of 1 ns (Thorlabs Det210) that monitored laser light partially transmitted through a turning mirror. The timing of the probe laser was monitored using a vacuum photodiode with an $\mathrm{Al}$ photo-cathode that collected light from the second diffracted order of the first diffraction grating. The time of arrival of the heating pulse to the target $(t=0)$ was experimentally confirmed by observing the time of occurrence of first changes in the interferograms near the target surface.

\section{INTERFEROMETRY RESULTS}

A sequence of interferograms describing the evolution of plasmas created by irradiating carbon semi-cylindrical cavities is shown in Fig. 2. The error in determining the electron density is estimated to be $\sim 5 \times 10^{18} \mathrm{~cm}^{-3}$, based on the resolution of 0.1 fringe shift for a $1 \mathrm{~mm}$ long plasma. The effect of lateral expansion along the axis of the plasma was determined by using the same laser line focus on a shorter $0.5 \mathrm{~mm}$ long target. The plasma densities resulting from the measurement of the two target lengths $(1$ and $0.5 \mathrm{~mm}$ ) differed by only about $10 \%$, which is within the measured shot to shot variation of the plasma. This shows that the lateral expansion does not significantly affect the measurements. Each 
interferogram in Fig. 2 corresponds to a different time measured with respect to the arrival of the $650 \mathrm{~mJ}$ heating pulse onto the target surface. Absorption of the SXRL probe beam can be noticed near the target surface due to the presence of dense cold plasma. This effect can be seen around $2.6 \mathrm{~ns}$ and increases in time as the plasma cools. This strong attenuation of the probe beam is caused by photoionization of low charge C ions (ie. C I, C II) and neutral atoms; resulting from plasma recombination, for which the photoionization potential is less than the $26.5 \mathrm{eV}$ energy of the photons in the probe beam. However, fringe visibility remains high farther away from the target and in the region where the density increase is observed. Furthermore, absorption does not preclude obtaining clear interference fringes at longer time delays such as $10.7 \mathrm{~ns}$.
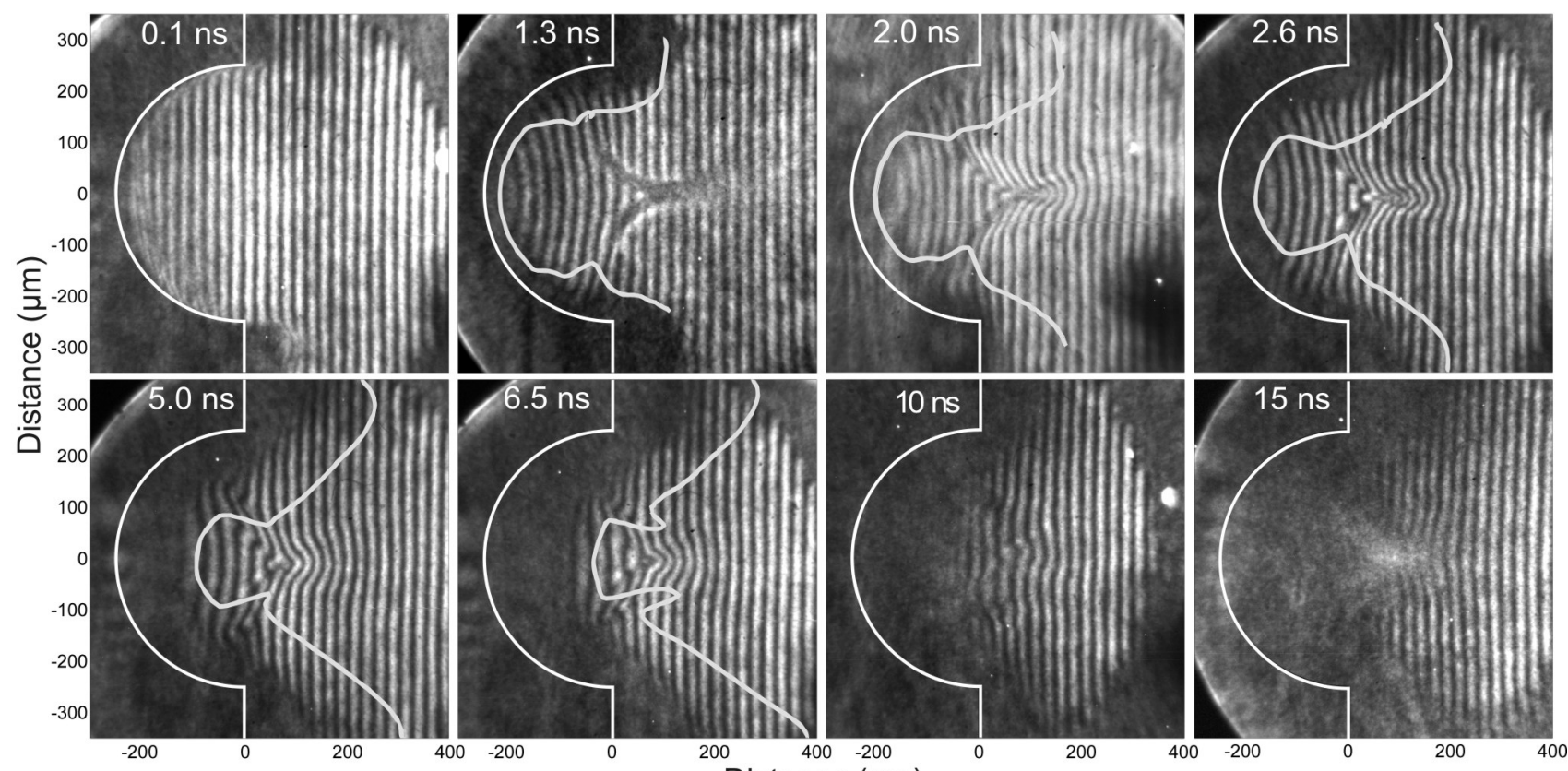

Distance $(\mu \mathrm{m})$

Figure 2. Sequence of soft x-ray laser interferograms describing the evolution of plasmas created by irradiating a semi-cylindrical carbon cavity with a 120 ps pulse at an intensity of $1.1 \times 10^{12} \mathrm{Wcm}^{-2}$. Time delays are measured with respect to the peak of the irradiation laser beam incident from the right. The large number of fringe shifts close to the axis of the groove is indicative of a high density region created by the converging plasma. The free electron approximation to the index of refraction is computed to be valid in the region to the right of the white line.

The first interferogram of the sequence was acquired at the time of the peak of the 120 ps heating pulse. Plasma absorption and fringe shifts are present in the region within $\sim 50 \mu \mathrm{m}$ of the target surface, indicating the early stage of the plasma expansion. In the $1.3 \mathrm{~ns}$ frame perturbed fringes are observed nearly everywhere within the cavity. Near the center of the groove we observe a region, shaped like a horizontal "Y" in which it is not possible to resolve the fringes; most likely due to strong refraction from steep density gradients and motion blurring from a rapid increase in density. In any case, this frame indicates that at $1.3 \mathrm{~ns}$ the plasma has already converged on axis and a fast density build-up is under way. In the $2 \mathrm{~ns}$ frame, a significant number of fringe shifts is observed in an oval shaped region near the axis, indicating the formation of a strongly pinched plasma. At $2.6 \mathrm{~ns}$ the fringes in this location remain highly perturbed, and absorption of the probe beam is observed near the surface of the target. The subsequent frame, at $5.0 \mathrm{~ns}$, shows a significant decrease in the number of fringe shifts near the axis. The shifting continues to decrease with time until $\sim 10 \mathrm{~ns}$, at which the fringe visibility inside the cavity is lost due to significant absorption of the probe beam. 


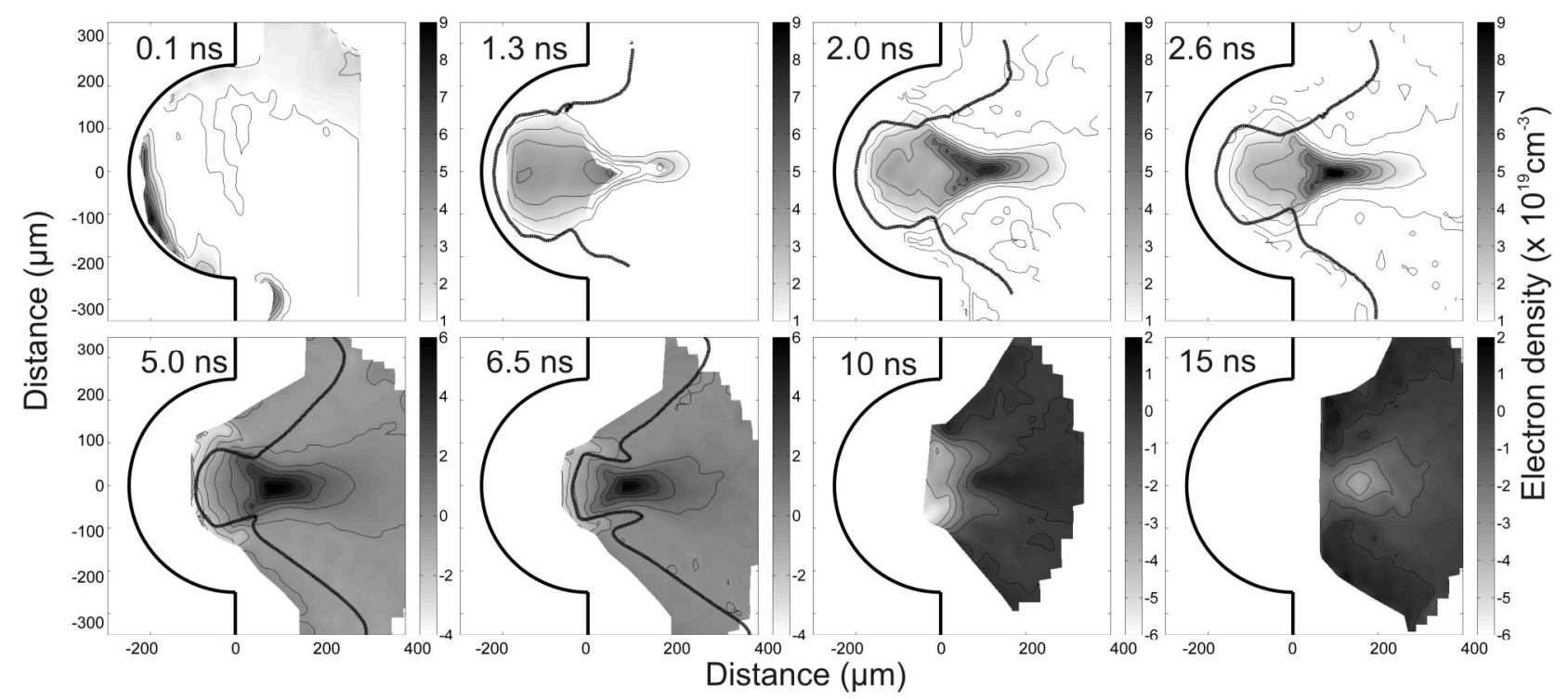

Figure 3. Measured carbon electron density maps corresponding to the interferograms shown in Fig. 2. An electron density scale is shown at the right with units of $10^{19} \mathrm{~cm}^{-3}$. The free electron approximation to the index of refraction is computed to be valid in the region to the right of the dark contour line.

The electron density maps resulting from the carbon plasma interferograms are shown in Fig. 3. The data shows that the plasma expands away from the surface of the target, converging in a small region just outside the axis of the cavity causing an abrupt increase in the electron density. This increase in plasma density is accompanied by a strong localized emission of radiation from that region, as shown in the time-integrated self emission image in the bottom right frame of Fig. 1, which was taken using a micro channel plate sensitive to UV/VUV. The increase in electron density develops as early as $1.3 \mathrm{~ns}$, reaching a measured maximum of $1.1 \times 10^{20} \mathrm{~cm}^{-3}$ at $2.4 \mathrm{~ns}$.

While it is possible to construct electron density maps from the measured fringe shifts, the commonly used assumption that only free electrons contribute to the refractive index is not valid for the colder regions of the carbon plasma. This reduces the region where accurate electron density maps can be obtained. In recent soft x-ray laser interferometry experiments we have observed anomalous fringe shifts caused by the contribution of bound electrons to the index of refraction [14]. For a probe laser wavelength of $46.9 \mathrm{~nm}$ anomalous fringe shifts were observed in tin, silver [15] and carbon [16] plasmas. In the case of the carbon plasmas it was found that the neutral atoms as well as singly and doubly ionized atoms can make significant contributions to the index of refraction. Therefore it is justifiable to assume that the free electrons are the main contribution to the index of refraction in regions where the mean degree of ionization is larger than 3. In practice only the presence of doubly charged ions is of concern, as significant concentrations of neutral atoms or singly ionized ions greatly attenuates the probe beam by photoionization. We used simulations conducted with the code HYDRA discussed in the next section to determine the plasma regions in which the free electron approximation can be considered valid and consequently the electron density maps accurate. The region to the right of the thin contour lines in Figs. 2 and 3 correspond to the simulated plasmas with mean degree of ionization larger than 3. In these regions, including the zone of high density build-up, the free electron approximation should be valid and therefore the electron density maps can be expected to be accurate. In addition, the region where the high density buildup was measured falls far from this boundary region and has a mean ionization of $\sim 5$. The regions to the left of the contour line, where the free electron approximation is not valid, present unphysical negative electron densities. The contour lines are not shown on the last two density maps because, due to the large abundance of lowly charged ions, the contribution of bound electrons make the free electron approximation invalid throughout most of the density map.

\section{SIMULATIONS AND DISCUSSION}

The converging carbon plasmas were modeled with HYDRA, a three-dimensional (3D) single fluid radiation hydrodynamic code that utilizes a structured mesh made up of hexahedrons[17, 18]. We chose to set the dimension of a unit cell along the target axis to equal the plasma length, effectively creating a $2 \mathrm{D}$ mesh. The good uniformity of the line focus, which overfills the target, helps to justify the assumption of uniformity along this dimension. 
Inverse Bremsstrahlung absorption is assumed to be the dominant laser deposition mechanism at our irradiation conditions. Radiation transport within the plasma was treated using multi-group diffusion techniques and tabulated opacities, and heat conduction was simulated using the conductivities of Lee and More[19]. The equations of state as well as the opacities are modeled using the Lawrence Livermore National Laboratory's LEOS library. The electron flux limiter was set to a value of 0.05. HYDRA is capable of running in Lagrangian mode, Eulerian mode or an Arbitrary Lagrangian Eulerian (ALE) hydrodynamic mode. An assortment of hydrodynamic mesh motion strategies, such as ALE, are available to help elude mesh tangling that commonly occurs when modeling a converging plasma. We chose a strategy in which the grid motion was set to be fully Lagrangian during the duration of the laser irradiation pulse. After the termination of the laser pulse we allowed the grid to move in the ALE mode. This strategy is well justified due to the fact that during the duration of the laser pulse the plasma blowoff does not reach the axial region, where severe mesh entanglement would occur. Therefore, the Lagrangian approach is most adequately used during this initial time interval. The ALE approach adaptively follows the expanding plasma and accurately reproduces shock waves automatically, the mesh is fine where density and its gradients are large and coarse when plasma rarifies.

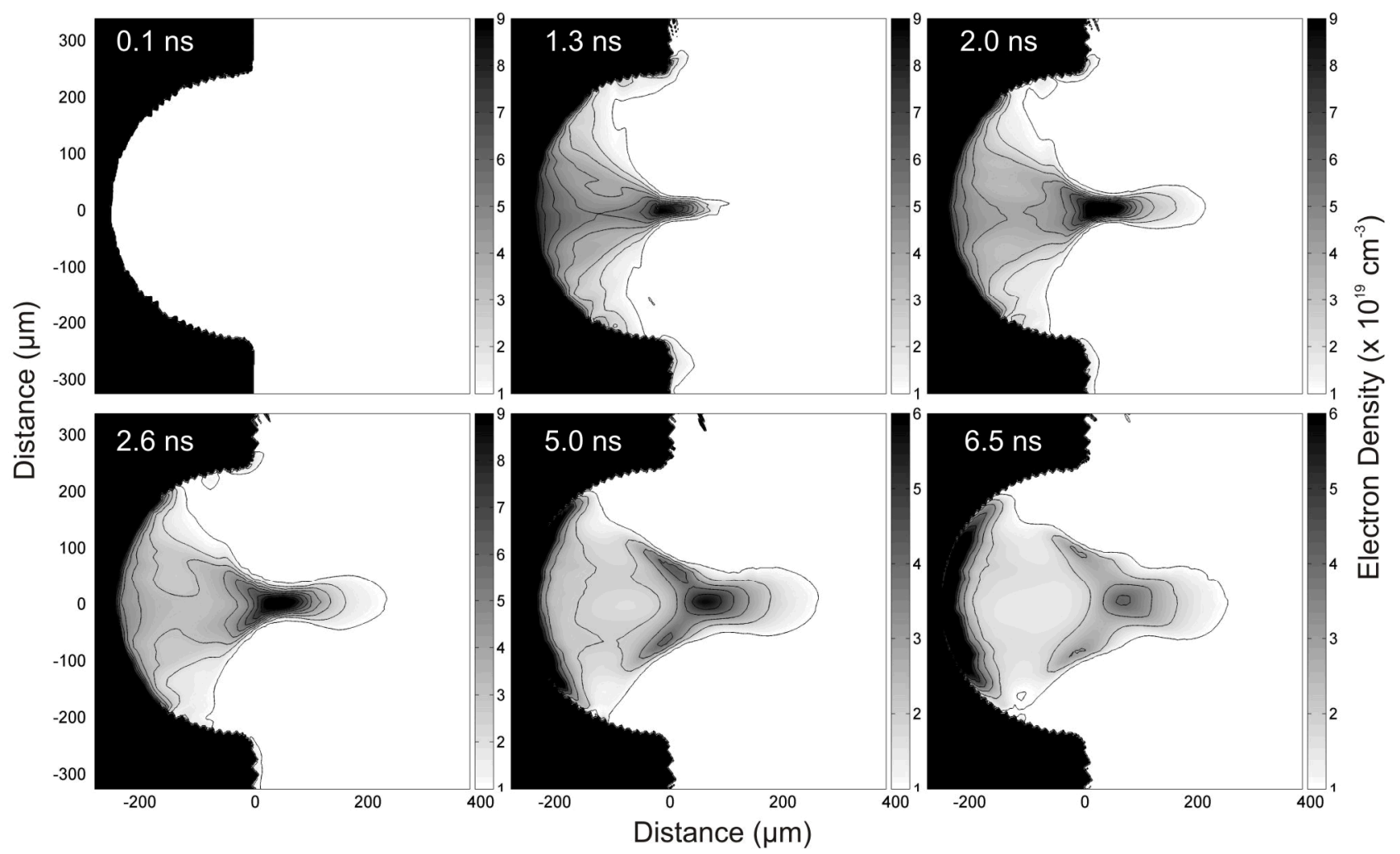

Figure 4. Simulated electron density maps for the carbon plasmas.

The simulated HYDRA electron density and average ionization maps are shown in Figs. 4 and 5 respectively. The computed electron density distributions compare well with the experimental data of Fig. 3 for the entire plasma region where the measurements are valid. As the simulated plasma expands, its low density plasma front begins to converge and collide as early as $800 \mathrm{ps}$ after the target irradiation. This early plasma convergence strengthens significantly during the next $500 \mathrm{ps,} \mathrm{forming} \mathrm{a} \mathrm{concentrated} \mathrm{high} \mathrm{density} \mathrm{plasma} \mathrm{near} \mathrm{the} \mathrm{axis} \mathrm{of} \mathrm{the} \mathrm{cavity} \mathrm{that} \mathrm{can} \mathrm{be}$ seen in the measurements at $1.3 \mathrm{~ns}$. Simulations and experimental results show that the plasma density peak forms in a region near the groove's axis. As more plasma arrives to this region the density peak moves away from the surface of the target, reaching a maximum value in a region just off the axis of the groove.

The effect of plasma converging towards a density build-up, unlike the focusing of light where photons do not interact with each other, is the result of increased repulsion in progressively denser plasma. Interpenetration occurs throughout the very early stages of the evolution when the density is small and ion speed is large. Moreover, interpenetration conditions exist during the onset of a density build-up, at a time when the plasma density is in the range of (1-3) $10^{19} \mathrm{~cm}^{-3}$ and the ion energy of the plasma edge expands with a speed of (1-3) $10^{7} \mathrm{~cm} / \mathrm{s}$, which corresponds to an ion kinetic energy between 5 and $30 \mathrm{keV}$. For these conditions the size of the plasma focal spot is small and the ion-ion elastic collision mean free path is much larger than this focal spot. As the plasma density increases the plasma rapidly 
becomes highly collisional and a single fluid hydrodynamic code like HYDRA, which does not account for interpenetration, can accurately simulate the plasma. The code also reveals that the electron temperature at this location initially increases to a value of $\sim 60 \mathrm{eV}$ and subsequently is replaced by colder plasma that expands from the back of the target. When the electron density near the axis reaches its peak the electron temperature is computed to be $\sim 40 \mathrm{eV}$.

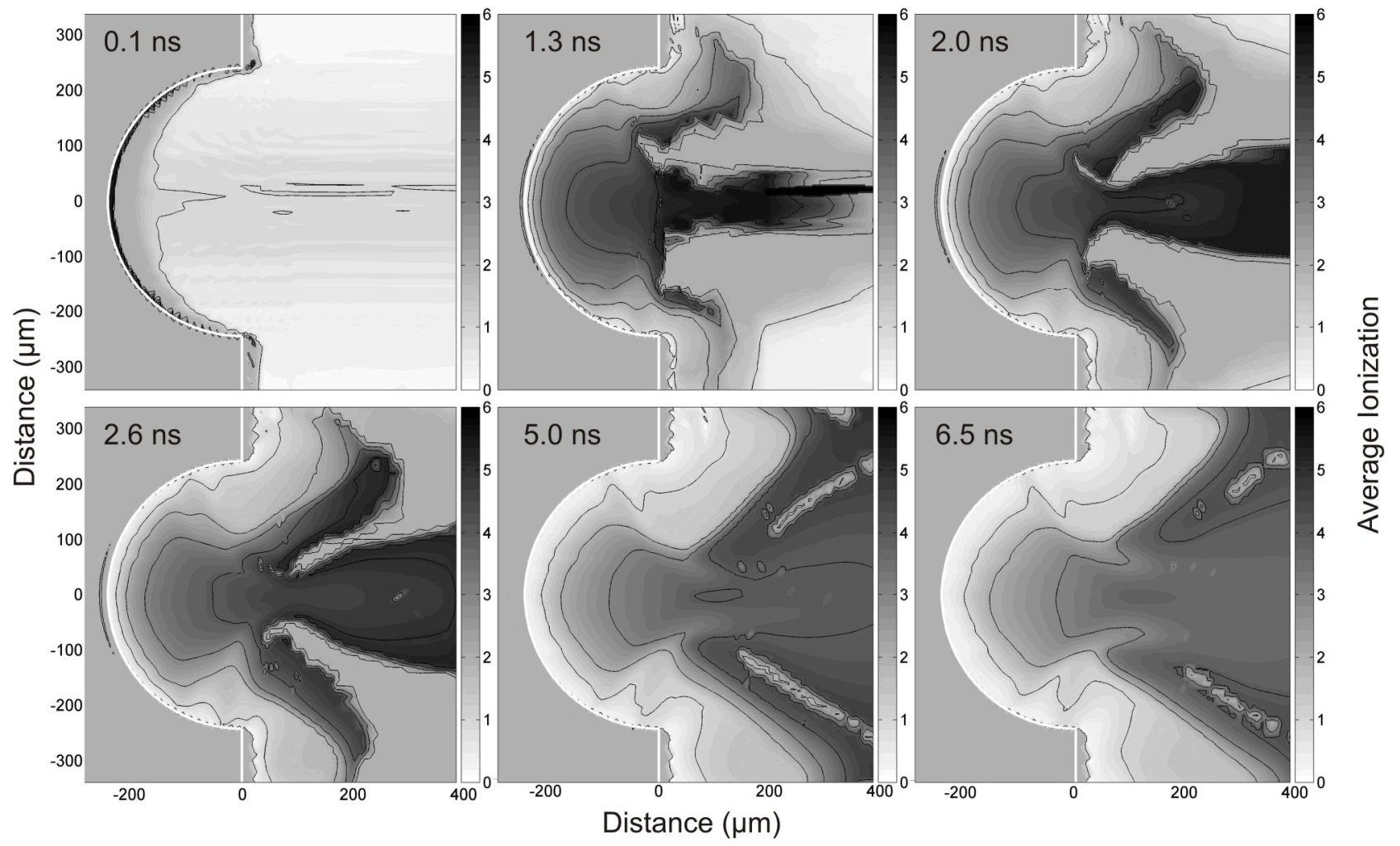

Figure 5. Simulated average ionization maps for the carbon plasmas.

The average ionization maps reveal regions near the corners of the target where ionization is slightly higher $\sim 4-$ 5. These regions increase in size until $\sim 4 \mathrm{~ns}$ subsequently cooling and recombining. The increase in ionization is attributed to the interaction of mass from the corners of the target which expands and collides into the central plasma blow off. Much like oblique shock heating, material from the target corners transfers its kinetic energy to thermal energy reheating side regions of the expanding blow off.

The possible role of interpenetration within the plasmas that converge in the focal region can be determined by computing the ion-ion collision mean free path accounting for the relative drift between ions. Decoster and Jones [20] have performed an analysis for counter-streaming plasmas yielding two ion-ion collision frequencies, one for dynamic friction, and another related to temperature equilibration [20], determined by the ratio of the corresponding plasma velocity and collision mean free path. The semi-cylindrical surface of our target requires the consideration of an angle dependent drift velocity in which the counter-streaming component of the drift velocity is minimized for small angles. Using the measured density data for the focal region and the simulated temperature and degree of ionization data from HYDRA the ion-ion collision mean free path for similar species can be computed to be less than $2.2 \mu \mathrm{m}$ which is small compared to the plasma dimensions. Further proof that interpenetration is not important at these conditions is the good agreement between the measured density maps and the results from the fluid code HYDRA which does not account for interpenetration.

To understand the relative contribution of plasma leaving different regions of the target surface to the density build on axis it is interesting to consider the laser irradiation profile and target geometry. The target irradiation intensity is maximum at the bottom of the groove, as a result of the combined effect of the nearly Gaussian profile of the laser that peaks at the center of the groove and the curvature of the target which presents a decreasing cross sectional area to the wings of the heating beam. Nearly $77 \%$ of the laser energy is deposited onto the target surface within the 74 degree angle segment corresponding to the heating beams FWHM as illustrated in Fig. 1. Therefore the plasma generated near the bottom of the groove is hotter and expands with a higher velocity, arriving to the focal region earlier. The expanding 
plasma generated in the upper and lower portions of the cavity are accelerated by significantly smaller pressure gradients and arrive on axis later in time.

To better determine the relative contribution of the plasma originating from different regions of the wall to the plasma density build-up, tracer particle trajectories were computed. Velocity vector fields were generated from HYDRA simulations at $10 \mathrm{ps}$ intervals that were then used to compute the trajectory of a set of test particles. The selected tracer particles are initially uniformly spaced along the curved surface and their position is modified in 10 ps steps by using the velocity fields. Figure 6 shows two velocity maps at two instants of time (1.0 and $2.5 \mathrm{~ns})$ in the evolution of the plasmas. The velocities of the plasma at the earlier times are mostly directed toward the center of the groove. The highest velocities, of $\sim 400 \mu \mathrm{m} / \mathrm{ns}$ are found there and correspond to the fastest particles that left the target early in the evolution of the plasma. In this region the velocity is redirected away from the target wall. This redirection is more evident in the later time frame, although the overall velocities inside the groove are slower. The fast particles have moved away from the wall, a distance of approximately $400 \mu \mathrm{m}$ no longer contributing to the density build-up.
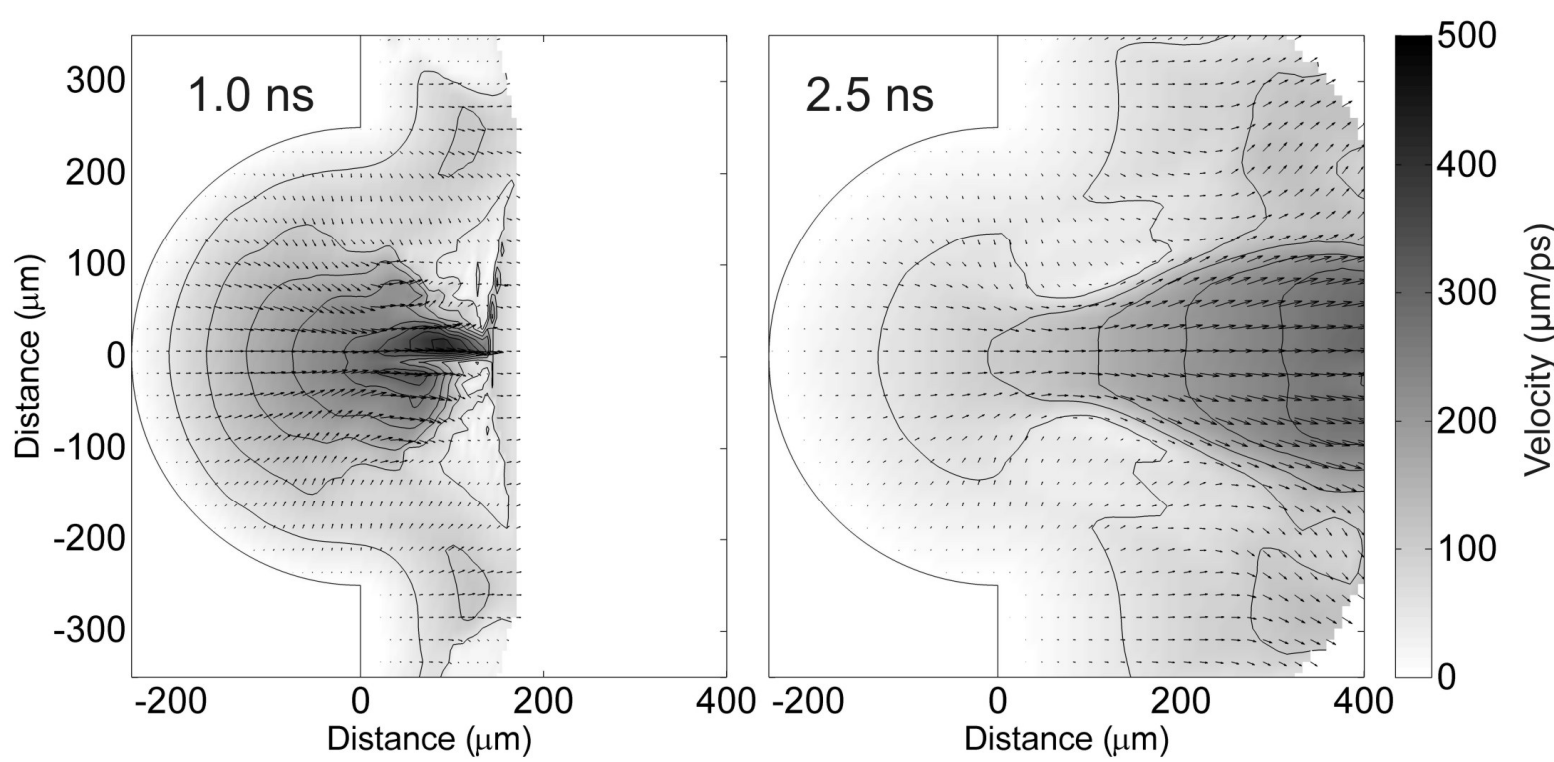

Figure 6. Velocity maps at two instants on the evolution of the plasmas simulated using the code HYDRA under the experimental conditions. The velocity gray scale is in micrometers/ns. The velocity maps were used construct trajectory plots of tracer particles shown in figure 7.

Figure 7 shows the trajectory of particles ablated at times of 70,140 , and $210 \mathrm{ps}$ with respect to the beginning of the 120 ps FWHM heating pulse. Each frame follows the tracer particles from the time they leave the surface (the darkest shade) up to $4 \mathrm{~ns}$ in the evolution (the lightest shade). Each alternating shade corresponds to a $500 \mathrm{ps}$ step in the particles trajectory. The graphs below the trajectory plots indicate the time with respect to the laser heating pulse at which the particles are created. The first particles to leave the target surface expand into vacuum accelerated outward by the increasing pressure gradients. The converging plasma emanating from within the 74 degree central region of the target at the time of the peak of the irradiation (70 ps frame) reaches the focus region in about $1.5 \mathrm{~ns}$, causing an abrupt increase in the plasma density. Plasma leaving the back of the target at the end of the pulse (140 ps frame) arrives to the focus region at $2 \mathrm{~ns}$ contributing significantly to the density build-up. In contrast, the tracer particles that start the evolution at target surface locations irradiated by a reduced intensity (larger angles) are noticeably slower and reach the focal region significantly later, near the end of the $4 \mathrm{~ns}$ evolution. Plasma radiation continues to ablate the target after the termination of the heating laser pulse. The trajectory plots that follow the particles which leave the target after the termination of the irradiation pulse ( $210 \mathrm{ps}$ frames) show this plasma moves significantly slower as a result of the colder temperature near the walls. Furthermore, plasma leaving the surface of the target at $210 \mathrm{ps}$ does not reach the focal region where a plasma build-up occurs during the first $4 \mathrm{~ns}$ of the evolution. At this time all the tracer particles leave the target surface with similar velocity, independently of their position due to a more uniform heating of the target surface. The density increase in the focal region is therefore dominantly caused by the convergence of plasma created at the bottom of the groove during the laser irradiation with additional contributions from plasma originating from the steeper wall of the cavity that arrives later and collides with little interpenetration. Fed mainly from the back of the target, the density increase occurs slightly off the axis of the cylinder due to the strong horizontal velocity component of this 
dominant plasma source. These trajectory plots also show a reduction in the vertical component of the expansion velocity, creating a narrowed stream of plasma that exits the cavity.
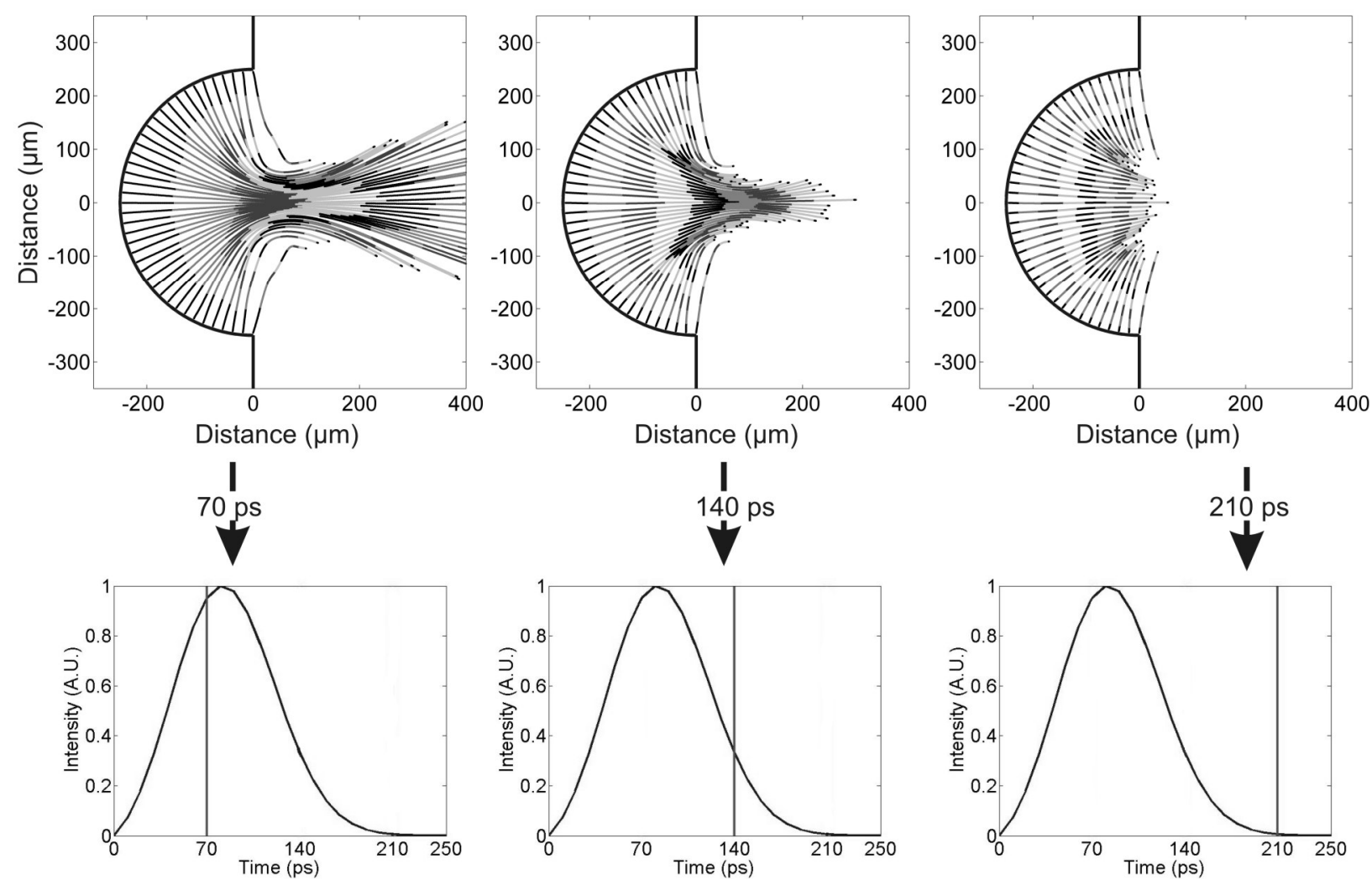

Figure 7. Plots revealing the $4 \mathrm{~ns}$ trajectory evolution of tracer particles leaving the target surface at three different time delays with respect to the beginning of the laser heating pulse. Alternating shades are used to represent time periods of $500 \mathrm{ps}$.

By following the tracer particles and computing the ionization of the plasma at each location of their trajectory we were able to determine a slight increase in ionization of $\sim 10 \%$ occurring while the plasma radially converges on axis. The increase in the ionization rate associated with the increased density in this region is compensated by the increase in collisional electron-ion recombination at the relatively small temperatures in this region.

\section{WORK IN PROGRESS}

Another soft x-ray interferometry study we are currently performing concerns the irradiation of $1 \mathrm{~mm}$ long aluminum "V shaped" cavities, resulting in the formation of jet-like structures. 


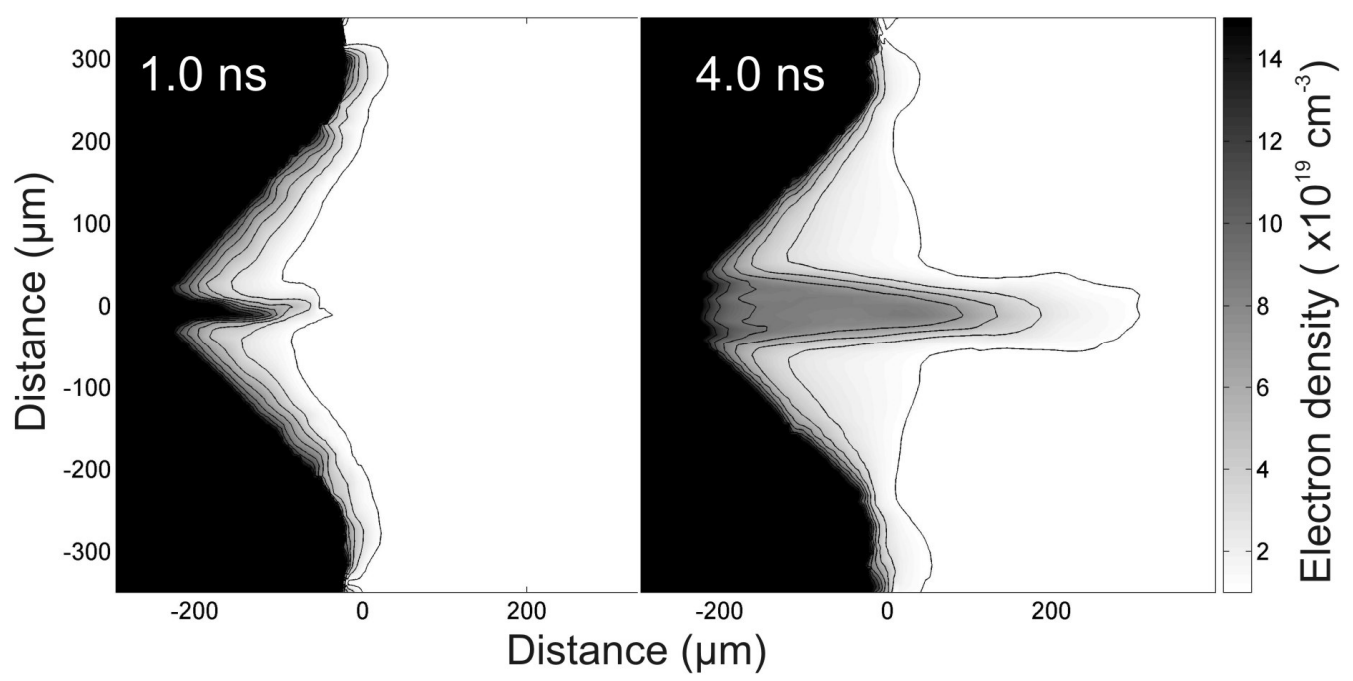

Figure 7. Preliminary HYDRA simulations of aluminum "V shaped" targets, irradiated under conditions similar to the semicylindrical targets of figure 2 . The creation of jet-like structures is observed.

Preliminary HYDRA simulations, shown above, have been performed on triangular cavities $250 \mu \mathrm{m}$ deep and $500 \mu \mathrm{m}$ opening with a $90^{\circ}$ angle at the tip, using laser irradiation conditions with a slightly higher peak intensity of $\sim 1.2 \times 10^{12}$ $\mathrm{Wcm}^{-2}$ than those of the semi-cylindrical cavities. These plasmas exhibit a very different behavior than the plasma expanding from the semi-cylidrical targets. Unlike, the semi-cylindrical targets which form a high density region near the axis of the cylinder, initial plasma expansion gives rise to the formation of a dense plasma plume along the plane of symmetry that can be seen in the simulations at $4 \mathrm{~ns}$.

\section{CONCLUSION}

We have studied the dynamics of plasmas generated by irradiation of semi-cylindrical carbon cavities with 120 ps duration optical laser pulses at an intensity of $1 \times 10^{12} \mathrm{~W} \mathrm{~cm}^{-2}$. Electron density maps obtained with the use of a soft $\mathrm{x}-$ ray interferometer show that the plasma expands away from the target surface, converging slightly off axis, where a bright high density plasma focus with an electron density higher than $1 \times 10^{20} \mathrm{~cm}^{-3}$ is formed. The combination of the interferometry results with 2-dimensional simulations conducted with the hydrodynamic code HYDRA enabled to construct a picture of the expansion and evolution of the plasmas. We also used the code simulations to compute the degree of ionization of the plasma, which plays an important role in determining the region where it is valid to compute the index of refraction based only on free electrons. This allowed us for the first time to determine the region of validity of density maps obtained from soft x-ray laser interferograms using this approximation.

Due to the uneven heating of the target a hotter plasma is created at the bottom of the groove. This hotter plasma expands faster reaching the axis of the groove first, where it converges and forms the dense plasma focus. This faster and hotter plasma is followed by the arrival of successively colder and slower plasma created by plasma radiationinduced ablation of the target after the termination of the laser pulse. The simulations show that the plasma collision in the focal region redirects the velocity narrowing the stream of plasma that leaves the cavity. Because the plasma is highly collisional, interpenetration is small, and the hydrodynamic code is capable of reliably computing the electron density distribution and resulting in a good agreement between the measured and simulated electron density maps. Collisions in the plasma focus region do not significantly increase the degree of ionization of the plasma. The plasma build-up occurring slightly off axis of the groove axis is instead mainly the result of the convergence of the plasma in a small region.

The results also illustrate that the combination of soft $\mathrm{x}$-ray laser interferometry with 2-dimensional hydrodynamic simulations is a powerful tool to study the dynamics of dense plasmas. Future work can combine the very rugged, high throughput diffraction grating interferometer used here with shorter wavelength x-ray lasers to allow the diagnostics of near-solid density plasmas at high energy density facilities.

\section{ACKNOWLEDGEMENTS}


The authors would like to thank M. Marinak for the helpful discussions regarding the HYDRA simulations. This research was sponsored by the National Nuclear Security Administration under the Stewardship Science Academic Alliances program through U.S. Department of Energy Research Grant \#DE-FG52-06NA26152, using facilities from the NSF ERC Center for Extreme Ultraviolet Science and Technology, award EEC-0310717. Part of this work was performed under the auspices of the U.S. Dept. of Energy by the University of California, Lawrence Livermore National Laboratory through the Institute of Laser Science and Application, under contract No. W-7405-Eng-48.

\section{REFERENCES}

1. B.A. Remington, R.P. Drake, H. Takabe, and D. Arnett, "A review of astrophysics experiments on intense lasers," $41^{\text {st }}$ Annual Meeting of the Division of Plasma Physics, Seattle, January 2000.

2. A.H. Bride, and R.A. Perley, Extragalactic Radio Jets. Annu. Rev. Astron. Astrophys., 1984. 22: p. 319.

3. H. Zinnecker, M.J. McCaughrean and J.T. Rayner, A symmetrically pulsed jet of gas from an invisible protostar in Orion. Nature, 1998. 394: p. 862.

4. J. Lindl, Phys. Plasmas 2, 3933 (1995).

5. T. R. Dittrich, S. W. Haan, M. M. Marinak, S. M. Pollaine, D. E. Hinkel, D. H. Munro, C. P. Verdon, G. L. Strobel, R. McEachern, R. C. Cook, C. C. Roberts, D. C. Wilson, P. A. Bradley, L. R. Foreman, and W. S. Varnum, Physics of Plasmas 6, 2164 (1999).

6. M. E. Jones, D. Winske, S. R. Goldman, R. A. Kopp, V. G. Rogatchev, S. A. Bel'kov, P. D. Gasparyan, G. V. Dolgoleva, N. V. Zhidkov, N. V. Ivanov, Y. K. Kochubej, G. F. Nasyrov, V. A. Pavlovskii, V. V. Smirnov, and Y. A. Romanov, Phys. Plasmas 3, 1096 (1996).

7. A. S. Wan, T. W. Barbee, R. C. Jr., P. Celliers, L. B. D. Silva, J. C. Moreno, P. W. Rambo, G. F. Stone, J. E. Trebes, and F. Weber, Physical Review E 55, 6293 (1997).

8. B. R. Benware, C. D. Macchietto, C. H. Moreno, and J. J. Rocca, Physical Review Letters 81, 5904 (1998).

9. J. Filevich, K. Kanizay, M. C. Marconi, J. L. A. Chilla, and J. J. Rocca, Optics Letters 25, 356 (2000).

10. J. Grava, M. Purvis, J. Filevich, M. C. Marconi, J. J. Rocca, J. Dunn, S. J. Moon, R. F. Smith, J. Nilsen, and V. N. Shlyaptsev, "Soft x-ray laser interferometry of colliding plasmas," SPIE conference on Soft X-ray lasers and Applications, San Diego, August 2005; Proc. SPIE Int. Soc. Opt. Eng. 5919, (2005).

11. C. D. Macchietto, B. R. Benware, and J. J. Rocca, Optics Letters 24, 1115 (1999).

12. Y. Liu, M. Seminario, F. G. Tomasel, C. Chang, J. J. Rocca, and D. T. Attwood, Physical Review A 63, 033802 (2001).

13. J. Filevich, J. J. Rocca, M. C. Marconi, R. F. Smith, J. Dunn, R. Keenan, J. R. Hunter, S. J. Moon, J. Nilsen, A. Ng, and V. N. Shlyaptsev, Applied Optics 43, 3938 (2004).

14. J. Filevich, J. J. Rocca, M. C. Marconi, S. J. Moon, J. Nilsen, J. H. Scofield, J. Dunn, R. F. Smith, R. Keenan, J. R. Hunter, and V. N. Shlyaptsev, Physical Review Letters 94, 035005 (2005).

15. J. Filevich, J. Grava, M. Purvis, M. C. Marconi, J. J. Rocca, J. Nilsen, J. Dunn, and W. R. Johnson, Physical Review E (Statistical, Nonlinear, and Soft Matter Physics) 74, 016404 (2006).

16. J. Filevich, J. Grava, M. Purvis, M. C. Marconi, J. J. Rocca, J. Nilsen, J. Dunn, and W. R. Johnson, Laser and Particle Beams 25, 1 (2007).

17. M. M. Marinak, G. D. Kerbel, N. A. Gentile, O. Jones, D. Munruo, S. Pollaine, T. R. Dittrich, and S. W. Haan, Physics of Plasmas 8, 2275 (2001).

18. M. M. Marinak, S. W. Haan, T. R. Dittrich, R. E. Tipton, and G. B. Zimmerman, Phys. Plasmas 5 (1998).

19. Y. T. Lee, and R. M. More, Phys. Fluids 27, 1273 (1984).

20. M. E. Jones, D. S. Lemons, R. J. Mason, V. A. Thomas, and D. Winske, Journal of Computational Physics 123, 169 (1996). 